\title{
Lower limb: from the Pacionian corpuscles to the acetabular version
}

\author{
Bruno Grignon $^{1} \cdot$ Fabrice Duparc ${ }^{2} \mathbb{D}$
}

Published online: 7 July 2021

(c) The Author(s), under exclusive licence to Springer-Verlag France SAS, part of Springer Nature 2021

By following a logical compilation from the ground support to the hip joint, the articles in this issue contribute to the anatomical aspects of human walking.

\section{Foot and ankle}

By fast-adapting afferent input from the sole, the Pacinian corpuscles are essential for walking. However, little is known about their distribution in the plantar subcutaneous tissue, especially in infants. A histological study carried out in eight near-term human feet revealed a highest number of Pacinian corpuscles in the anterior foot and a decrease toward the heel, different from a mini-version of the adult, suggesting an infant-specific function, with initial walking pattern using the anterior foot without keel contact.

As well, the flexor hallucis longus and flexor digitorum longus tendons play an important role for walking. They are also frequently used in various surgery procedures, such as for reconstructive purposes, for posterior tibial dysfunction, or Achilles tendinopathy, among others. In this regard, the chiasma plantare formation is a precious surgical landmark with numerous variants that must be known preoperatively. This formation may be accurately evaluated by sonography, as was demonstrated by the sono-anatomical correlations performed on 22 ankles of adult cadavers presented in this issue. Other intertendinous connections (between the flexor digitorum brevis and longus) may also be assessed

Fabrice Duparc

fabrice.duparc@univ-rouen.fr

Bruno Grignon

b.grignon@chu-nancy.fr

1 Department of Anatomy, Faculty of Medicine, University of Lorraine, Avenue de la Forêt de Haye, 54505 Vandœuvre-Lès-Nancy, France

2 Department of Anatomy, Faculty of Medicine-Pharmacy, Rouen-Normandy University, 22 Boulevard Gambetta, 76183 Rouen, France by sonography, as is the case in the follow-up of flexor digitorum longus tenotomies performed for treatment for claw toes.

On the dorsum of the foot, the extensor hallucis longus muscle may present frequent variations that may be significant for both diagnostic accuracy and surgical planning, in particular for tendon transfer or graft. They can also contribute to the development of hallux valgus deformity. Special attention has been paid to this muscle and its variations in this issue through three different papers using cadaveric dissection, which showed important individual and ethnic differences.

The os trigonum, the second most common accessory bone of the foot may be of clinical importance since it can be involved in impingement syndrome, with the possible role of its mobility. An MR study of the ligaments of the os trigonum is presented in this issue, pointing out numerous connections with all posterior ankle structures, the potential clinical implications of which are discussed.

The twisted structure of the calcaneal tendon is known for a long time in adults, but had never been investigated in fetuses. In addition to its physiological role, this pattern is of particular interest for surgical techniques for lengthening the calcaneal tendon. The dissection of 36 human fetuses in the second trimester fixed in formaldehyde revealed a structure of the calcaneal tendon similar to that in the adult, made up of twisted subtendons.

Indirectly participating to the constitution of the calcaneal tendon, a third head of the gastrocnemius may be encountered with a frequency of 1.9-5.5\% among various populations and should be distinguished from a double plantaris muscle, as is discussed in a study pointing out the importance of the innervation in this regard.

\section{Knee}

The anterolateral ligament of the knee was in the limelight a few years ago. However, discrepancy remains as regards its presence in fetuses. A mesoscopic analysis carried out 
in 40 human fetuses knee joints at mean age 34 weeks is presented in this issue and concludes that this ligament is not a solid structure in fetuses but could be a deep layer of the ilio-tibial tract.

In spite of remaining controversies about the exact role of the meniscus in knee stability, the influence of their posterior horns on the posterior tibial bone slope has been suggested for many years and confirmed by a study using MR images in 325 patients reported in this number.

The vascular supply of the proximal fibula may have important clinical implications such as vascularized proximal fibular transfer, a reconstruction method for joints and growing bones. The results of a computed tomographic angiography combined with anatomical dissection in 20 fresh cadaveric knees are described and discussed in this issue.

\section{Hip}

The symmetry of the hip joint is of the highest practical importance in order to assess the template of total hip arthroplasty since the uninjured side could be used for the preoperative plan of the other hip. The study of 224 patients using $\mathrm{X}$-ray and CT scan confirmed this hypothesis in all cases.

The relationship between pelvic incidence and anatomical acetabular anteversion studied by means of $110 \mathrm{CT}$ scans is also presented and discussed in this issue.

\section{Infragluteal fold}

In all times and cultures, the gluteal region has played an important role in esthetics and, as a result, presents today a major interest in plastic surgery. However, one of its key-component, the infragluteal fold, has not yet been clearly defined. By means of MR imaging of volunteers and dissection of fresh cadavers, a here presented study revealed the infragluteal fold as a connectival fibrous band extending from the ramus of the ischium, the coccyx and the apex of sacrum, without significant differences in measurement between volunteer and cadaver group.

\section{Iliac vein}

Finally, just above the lower limb, qualitative and quantitative characteristics of compression of the iliac veins between the iliac arteries and the lumbosacral vertebrae have been investigated by means of contrast-enhanced CT scan in 195 subjects, providing a novel classification that can be useful to predict which patients will benefit from interventional therapy.

Wishing you a valuable reading.

Publisher's Note Springer Nature remains neutral with regard to jurisdictional claims in published maps and institutional affiliations. 